\title{
Character varieties of double twist links
}

\author{
KATHLEEN L PETERSEN \\ ANH T TRAN
}

\begin{abstract}
We compute both natural and smooth models for the $\mathrm{SL}_{2}(\mathbb{C})$ character varieties of the two-component double twist links, an infinite family of two-bridge links indexed as $J(k, l)$. For each $J(k, l)$, the component(s) of the character variety containing characters of irreducible representations are birational to a surface of the form $C \times \mathbb{C}$, where $C$ is a curve. The same is true of the canonical component. We compute the genus of this curve, and the degree of irrationality of the canonical component. We realize the natural model of the canonical component of the $\mathrm{SL}_{2}(\mathbb{C})$ character variety of the $J(3,2 m+1)$ link as the surface obtained from $\mathbb{P}^{1} \times \mathbb{P}^{1}$ as a series of blow-ups.
\end{abstract}

$57 \mathrm{M} 25 ; 57 \mathrm{~N} 10,14 \mathrm{~J} 26$

\section{Introduction}

Given a complete orientable finite-volume hyperbolic 3-manifold with cusps, the $\mathrm{SL}_{2}(\mathbb{C})$ character variety of $M, X(M)$, is an affine complex algebraic set associated to representations $\pi_{1}(M) \rightarrow \mathrm{SL}_{2}(\mathbb{C})$. Thurston [14] showed that any irreducible component of such a variety containing the character of a discrete faithful representation has complex dimension equal to the number of cusps of $M$. Such components are called canonical components and are denoted $X_{0}(M)$. Character varieties have been fundamental tools in studying the topology of $M$ (we refer the reader to Shalen [13] for more), and canonical components encode a wealth of topological information about $M$, including containing subvarieties associated to Dehn fillings of $M$ and identifying boundary slopes of essential surfaces; see Culler and Shalen [3].

We consider the two-component double twist links $J(k, l)$ and compute the character varieties of their complements in $S^{3}$. As pictured in Figure 1, the integers $k$ and $l$ determine the number of half-twists in the boxes; positive numbers correspond to right-handed twists and negative numbers correspond to left-handed twists. The link $J(k, l)$ is a two-component link when $k l$ is odd and a knot when $k l$ is even. Macasieb, Petersen and van Luijk [8] determined and analyzed character varieties of the $J(k, l)$ knots. In this paper, we extend this work to the two-component $J(k, l)$ links. These 

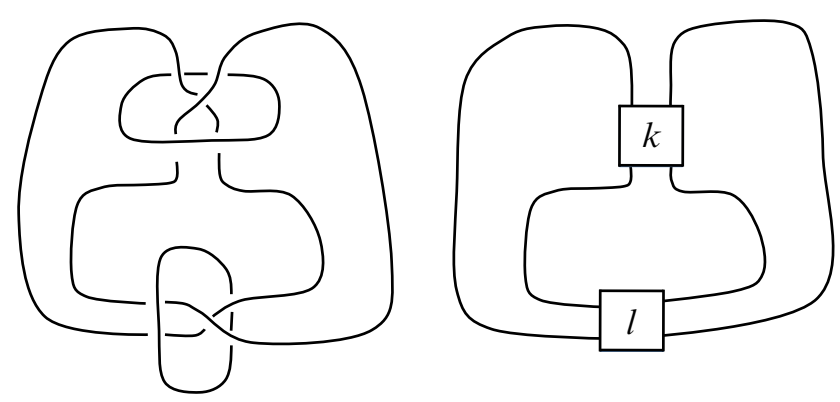

Figure 1: The link $J(k, l)$ is the result of $-1 / k$ and $-1 / l$ surgery on the four-component link pictured on the left.

are hyperbolic exactly when $|k|$ and $|l|$ are greater than one; the $J( \pm 1, l)=J(l, \pm 1)$ links are torus links. We will now exclusively consider the hyperbolic $J(k, l)$ links.

In Definition 3.5 we define the Chebyshev polynomials $S_{j}$ which are used throughout the paper. Our first theorem establishes natural models for the $\mathrm{SL}_{2}(\mathbb{C})$ character varieties of the double twist links. With $\pi_{1}(k, l)=\pi_{1}\left(S^{3}-J(k, l)\right)$, let $X_{\text {irr }}(k, l)$ denote the closure of the set of all irreducible characters $\chi_{\rho}$ of representations $\rho: \pi_{1}(k, l) \rightarrow \mathrm{SL}_{2}(\mathbb{C})$. Let $X_{0}(k, l)$ denote a canonical component. In fact, a consequence of this work is that for a given double twist link, there is only one canonical component. For this natural model, we use the presentation for $\pi_{1}(k, l)$ in Section 3 with $x=\chi_{\rho}(a), y=\chi_{\rho}(b)$ and $z=\chi_{\rho}\left(a b^{-1}\right)$. The vanishing set of the characters of reducible representations $\pi_{1}(k, l) \rightarrow \mathrm{SL}_{2}(\mathbb{C})$ is well-known and is given by

$$
x y z+4-x^{2}-y^{2}-z^{2}
$$

in $\mathbb{C}^{3}(x, y, z)$. These are all characters of abelian representations.

Theorem 1.1 Let $k=2 m+1$ and $l=2 n+1$. A natural model for the algebraic set $X_{\mathrm{irr}}(k, l)$ is the vanishing set of

$$
S_{n}(t) S_{m-1}(z)-S_{n-1}(t) S_{m}(z)
$$

in $\mathbb{C}^{3}(x, y, z)$, where

$$
\begin{aligned}
t=\left(x S_{m}(z)-y S_{m-1}(z)\right)\left(y S_{m}(z)-x\right. & \left.S_{m-1}(z)\right) \\
& -z\left(S_{m}^{2}(z)+S_{m-1}^{2}(z)\right)+4 S_{m}(z) S_{m-1}(z) .
\end{aligned}
$$

The expression $t$ is the trace of $\rho(\beta)$, with the loop $\beta$ as pictured in Figure 2. In terms of the presentation for the fundamental group in Section 3, the loop $\beta$ corresponds to the word $w_{k}$. 
Our next theorem establishes smooth models for these algebraic sets.

Theorem 1.2 Let $k=2 m+1$ and $l=2 n+1$. The algebraic set $X_{\text {irr }}(k, l)$ is birational to $C(k, l) \times \mathbb{C}$, where the curve $C(k, l) \subset \mathbb{C}^{2}(t, z)$ is given by

$$
C(k, l)=\left\{S_{n}(t) S_{m-1}(z)-S_{n-1}(t) S_{m}(z)=0\right\} .
$$

If $k \neq l$ then $C(k, l)$ is smooth and irreducible as considered in $\mathbb{P}^{1}(t) \times \mathbb{P}^{1}(z)$, and $X_{0}(k, l)=X_{\text {irr }}(k, l)$ is birational to $C(k, l) \times \mathbb{C}$.

The curve $C(3,3)=C(-3,-3)$ is given by $t=z$. If $k=l$ and $|l|>3$ then $C(l, l)$ is the union of exactly two components: $C_{0}(l, l)$, given by $t=z$, and $C_{1}(l, l)$, the scheme-theoretic complement of $C_{0}(l, l)$ in $C(l, l)$. Both are smooth and irreducible as considered in $\mathbb{P}^{1}(t) \times \mathbb{P}^{1}(z)$. The algebraic set $X_{\text {irr }}(k, l)$ is given by the union $X_{0}(l, l) \cup X_{1}(l, l)$, where $X_{0}(l, l)$ is birational to $C_{0}(l, l) \times \mathbb{C}$ and $X_{1}(l, l)$ is birational to $C_{1}(l, l) \times \mathbb{C}$.

We next compute some invariants of these algebraic sets. Since $X_{\text {irr }}(k, l)$ is birational to the product of a curve $C(k, l)$ and $\mathbb{C}$, we compute the genus of this curve.

Theorem 1.3 Let $k=2 m+1$ and $l=2 n+1$ with $|k|,|l|>1$. When $k \neq l$ the genus of $C(k, l)$ is

$$
\left(\left\lfloor\frac{|k|}{2}\right\rfloor-1\right)\left(\left\lfloor\frac{|l|}{2}\right\rfloor-1\right) .
$$

The genus of $C_{0}(l, l)$ is zero, and when $|l|>3$ the genus of $C_{1}(l, l)$ is $\left(\left\lfloor\frac{|l|}{2}\right\rfloor-2\right)^{2}$.

The degree of irrationality of an irreducible $n$-dimensional complex algebraic set $X$ is defined to be the minimal degree of any rational map from $X$ to a dense subset of $\mathbb{C}^{n}$. This is denoted $\gamma(X)$ and is a birational invariant. When $X$ is a curve this is called the gonality of $X$. See Petersen and Reid [11] for a discussion on how gonality and genus behave in families of Dehn fillings. In light of this, since $J(k, l)$ is $-1 / k$ and $-1 / l$ filling of the four-component link in Figure 1, we compute the degree of irrationality of the surfaces $X_{0}(k, l)$ and $X_{1}(l, l)$.

Theorem 1.4 Let $k=2 m+1$ and $l=2 n+1$. The degree of irrationality of $X_{0}(k, l)$ is $\min \left\{\left\lfloor\frac{|k|}{2}\right\rfloor,\left\lfloor\frac{|l|}{2}\right\rfloor\right\}$ when $k \neq l$. The degree of irrationality of $X_{0}(l, l)$ is 1 , and when $|l|>3$ the degree of irrationality of $X_{1}(l, l)$ is $\left\lfloor\frac{|l|}{2}\right\rfloor-1$.

Finally, we study the $J(3,2 m+1)$ links realizing $X_{0}(3,2 m+1)$ as a series of blow-ups of $\mathbb{P}^{1} \times \mathbb{P}^{1}$ and show the following. 
Theorem 1.5 The desingularization of the natural model for the canonical component of the $\mathrm{SL}_{2}(\mathbb{C})$ character variety of the double twist link $J(3,2 m+1)$ is the conic bundle over the projective line $\mathbb{P}^{1}$ which is isomorphic to the surface obtained from $\mathbb{P}^{1} \times \mathbb{P}^{1}$ by repeating a one-point blow-up $9 m$ times if $m \geq 1$, and $-(6+9 m)$ times if $m \leq-2$. Equivalently, it is isomorphic to the surface obtained from $\mathbb{P}^{2}$ by repeating a one-point blow-up $1+9 m$ times if $m \geq 1$, and $-(5+9 m)$ times if $m \leq-2$.

Remark 1.6 For $m \geq 1$, the link $J(3,2 m+1)$ is obtained by $1 / m$ Dehn surgery on the magic manifold. Hence Theorem 1.5 confirms Conjecture 3.1.3 in Landes' thesis [7].

Acknowledgement This work was partially supported by a grant from the Simons Foundation (number 209226 to Kathleen Petersen).

\section{Character varieties}

We will define our notation, but refer the reader to [8] for a detailed discussion of character varieties. Let $M$ be a complete finite-volume hyperbolic 3-manifold. The $\mathrm{SL}_{2}(\mathbb{C})$ character variety of $M$ is the set of all characters of representations $\rho: \pi_{1}(M) \rightarrow \mathrm{SL}_{2}(\mathbb{C})$. The character associated to $\rho$ is $\chi_{\rho}: \pi_{1}(M) \rightarrow \mathbb{C}$, defined by $\chi_{\rho}(\gamma)=\operatorname{tr} \rho(\gamma)$.

Let $X(M)$ denote the $\mathrm{SL}_{2}(\mathbb{C})$ character variety, that is

$$
X(M)=\left\{\chi_{\rho} \mid \rho: \pi_{1}(M) \rightarrow \mathrm{SL}_{2}(\mathbb{C})\right\} .
$$

The characters of reducible representations themselves form an algebraic set, which is a subset of $X(M)$. We will call this set $X_{\text {red }}(M)$. The closure of the set of characters of irreducible representations will be denoted by $X_{\text {irr }}(M)$. Any irreducible component of $X(M)$ which contains the character of a discrete faithful representation is contained in $X_{\text {irr }}(M)$ and is called a canonical component and denoted $X_{0}(M)$.

Thurston [14] showed that the complex dimension of any canonical component equals the number of cusps of $M$. Canonical components encode much of the topology of $M$, often seen through the trace functions. Canonical components containing subvarieties corresponding to Dehn fillings of $M$ and their ideal points can be used to determine essential surfaces in $M$ (see [3]).

When $M$ has only one cusp $X_{0}(M)$ is a curve. Several infinite families of these have been studied. (See $[1 ; 8 ; 16]$, for explicit computations. See [10] and [2] for examples of families of manifolds with many components in their character varieties.) When $M$ has at least two cusps the algebraic geometry becomes more demanding, and only a 
few solitary examples have been computed. Landes [6; 7] computed a smooth model for the canonical component of the $\mathrm{SL}_{2}(\mathbb{C})$ character variety of the complement of the Whitehead link, a two-component link. (She explicitly showed that it is a rational surface homeomorphic to the projective plane blown up at 10 points.) Harada [5] computed the character varieties of the four arithmetic two-bridge link complements (including the Whitehead link and the figure- 8 knot). Our computation of the character varieties of the double twist links is the first result to compute character varieties for infinitely many 3-manifolds with two cusps.

\section{Double twist links}

Let $J(k, l)$ be the double twist link indicated in the right-hand side of Figure 1. This link is $-1 / k$ and $-1 / l$ filling on two components of the four-component link shown in the left-hand side of Figure 1. This is a knot when $k l$ is even and a two-component link when $k l$ is odd. The link $J(k, l)$ corresponds to the continued fraction $[k,-l]$. It is hyperbolic, unless $|k|$ or $|l|$ is 1 . Let $X(k, l)$ denote the $\mathrm{SL}_{2}(\mathbb{C})$ character variety of $S^{3}-J(k, l)$.

In [8] the character varieties of the $J(k, l)$ knots were computed. We now consider the $J(k, l)$ links with two components, so both $k$ and $l$ are odd. Suppose $k=2 m+1$ and $l=2 n+1$. The link group of $J(k, l)$ is $\pi_{1}(k, l)=\pi_{1}\left(S^{3}-J(k, l)\right)$ and has presentation

$$
\pi_{1}(k, l)=\left\langle a, b \mid a w_{k}^{n} b=w_{k}^{n+1}\right\rangle,
$$

where $w_{k}=\left(a b^{-1}\right)^{m} a b\left(a^{-1} b\right)^{m}[8]$.

Definition 3.1 Let $F_{a, b}=\langle a, b\rangle$ be the free group on two letters $a$ and $b$. For a word $u$ in $F_{a, b}$ let $\overleftarrow{u}$ denote the word obtained from $u$ by writing the letters in $u$ in reversed order.

We begin by simplifying the presentation of the link group.

Lemma 3.2 With $w_{k}=\left(a b^{-1}\right)^{m} a b\left(a^{-1} b\right)^{m}$ and $r=w_{k}^{n}\left(a b^{-1}\right)^{m}$, we have

$$
\pi_{1}(k, l)=\langle a, b \mid r=\overleftarrow{r}\rangle
$$

Proof We can rewrite the presentation of $\pi_{1}(k, l)$ as

$$
\begin{aligned}
\pi_{1}(k, l) & =\left\langle a, b \mid a w_{k}^{n} b=\left(a b^{-1}\right)^{m} a b\left(a^{-1} b\right)^{m} w_{k}^{n-1}\left(a b^{-1}\right)^{m} a b\left(a^{-1} b\right)^{m}\right\rangle \\
& =\left\langle a, b \mid w_{k}^{n}=\left(b^{-1} a\right)^{m} b\left(a^{-1} b\right)^{m} w_{k}^{n-1}\left(a b^{-1}\right)^{m} a\left(b a^{-1}\right)^{m}\right\rangle \\
& =\left\langle a, b \mid w_{k}^{n}\left(a b^{-1}\right)^{m}=\left(b^{-1} a\right)^{m} b\left(a^{-1} b\right)^{m} w_{k}^{n-1}\left(a b^{-1}\right)^{m} a\right\rangle .
\end{aligned}
$$


Let $c=\left(a b^{-1}\right)^{m} a$ and $d=b\left(a^{-1} b\right)^{m}$. Then $w_{k}=c d$. It follows that

$$
b\left(a^{-1} b\right)^{m} w_{k}^{n-1}\left(a b^{-1}\right)^{m} a=d(c d)^{n-1} c=(d c)^{n}=\overleftarrow{(c d)^{n}}=\overleftarrow{w_{k}^{n}}
$$

Hence

$$
\begin{aligned}
\pi_{1}(k, l) & =\left\langle a, b \mid w_{k}^{n}\left(a b^{-1}\right)^{m}=\overleftarrow{\left(a b^{-1}\right)^{m}} \overleftarrow{w_{k}^{n}}\right\rangle \\
& =\left\langle a, b \mid w_{k}^{n}\left(a b^{-1}\right)^{m}=\overleftarrow{w_{k}^{n}\left(a b^{-1}\right)^{m}}\right\rangle
\end{aligned}
$$

Since $r=w_{k}^{n}\left(a b^{-1}\right)^{m}$, the lemma follows.

With coordinates $x=\operatorname{tr} \rho(a), y=\operatorname{tr} \rho(b)$ and $z=\operatorname{tr} \rho\left(a b^{-1}\right)$, the character variety of the free group $F_{a, b}$ is isomorphic to $\mathbb{C}^{3}[x, y, z]$ by the Fricke-Klein-Vogt theorem [4; 17]. Consider a word $u$ in $F_{a, b}$. Define the polynomial $P_{u} \in \mathbb{C}[x, y, z]$ to be $P_{u}(x, y, z)=\operatorname{tr} \rho(u)$. It follows that for every word $u$ in $F_{a, b}$ the polynomial $P_{u}$ is the unique polynomial such that for any representation $\rho: F_{a, b} \rightarrow \mathrm{SL}_{2}(\mathbb{C})$ we have $\operatorname{tr} \rho(u)=P_{u}(x, y, z)$.

We now consider representations $\rho: \pi_{1}(k, l) \rightarrow \mathrm{SL}_{2}(\mathbb{C})$. By Lemma 3.2 the group $\pi_{1}(k, l)$ has a presentation with two generators and one relation and therefore is a quotient of $F_{a, b}$. First, we establish some notation which we will use throughout the manuscript.

Definition 3.3 Let $k=2 m+1$ and $l=2 n+1$. For $\rho: \pi_{1}(k, l) \rightarrow \mathrm{SL}_{2}(\mathbb{C})$ define

$$
x=\operatorname{tr} \rho(a), \quad y=\operatorname{tr} \rho(b) \quad \text { and } \quad z=\operatorname{tr} \rho\left(a b^{-1}\right),
$$

and for a word $u$ in $F_{a, b}$ define the polynomial $P_{u}(x, y, z)=\operatorname{tr} \rho(u) \in \mathbb{C}[x, y, z]$. Further, let $t=P_{w_{k}}$ and

$$
\varphi(x, y, z)=P_{r a b}-P_{\overleftarrow{r} a b}
$$

For every representation $\rho: \pi_{1}(k, l) \rightarrow \mathrm{SL}_{2}(\mathbb{C})$, we consider $x, y$ and $z$ as functions of $\rho$. Using the presentation above for $\pi_{1}(k, l)$ with two generators and one relation, we conclude that $P_{r a b}=P_{\overleftarrow{r} a b}$, which is simply $\varphi(x, y, z)=0$, in $X(k, l)$. In fact, by [16, Theorem 1] $X(k, l)$ is exactly the zero set of $\varphi(x, y, z)$. (See also [12, Theorem 2.1]). Moreover, because of the format of the defining word, $P_{\overleftarrow{r} a b}=P_{b a r}$ [16, Theorem 1]. (That is, these polynomials in $\mathbb{C}^{3}[x, y, z]$ are identical.) Therefore, $\varphi(x, y, z)=P_{r a b}-P_{b a r}$. We summarize this discussion in the following proposition.

Proposition 3.4 The polynomial $\varphi(x, y, z)$ is given by $P_{r a b}-P_{b a r}$. The character variety $X(k, l)$ is the zero set of $\varphi(x, y, z)$ in $\mathbb{C}^{3}(x, y, z)$. 
We wish to obtain a nice format for $\varphi$. We introduce a family of Chebyshev polynomials, often called the Fibonacci polynomials, that will be essential to our computation of $\varphi$. (These are slightly different polynomials than were used in [8]; the indices are shifted by one.)

Definition 3.5 Let $S_{j}(\omega)$ be the Chebyshev polynomials defined by

$$
S_{0}(\omega)=1, \quad S_{1}(\omega)=\omega \quad \text { and } \quad S_{j+1}(\omega)=\omega S_{j}(\omega)-S_{j-1}(\omega)
$$

for all integers $j$.

It is elementary to verify the following lemmas.

Lemma 3.6 With $\omega=\sigma+\sigma^{-1}$ we have

$$
S_{j}(\omega)=\frac{\sigma^{j+1}-\sigma^{-j-1}}{\sigma-\sigma^{-1}} .
$$

The degree of $S_{j}$ is $j$ if $j>-1$ and $-j-2$ if $j<-1$.

Lemma 3.7 Suppose the sequence $\left\{f_{j}\right\}_{j \in \mathbb{Z}}$ satisfies the recurrence relation $f_{j+1}=$ $\omega f_{j}-f_{j-1}$ for all integers $j$. Then $f_{j}=S_{j}(\omega) f_{0}-S_{j-1}(\omega) f_{-1}$.

The following lemma can be verified by using Lemma 3.6.

Lemma 3.8 We have

(a) $S_{j}^{2}(\omega)+S_{j-1}^{2}(\omega)-\omega S_{j}(\omega) S_{j-1}(\omega)=1$,

(b) $S_{j}^{2}(\omega)-S_{j-1}^{2}(\omega)=S_{2 j}(\omega)$,

(c) $S_{m-1}(\omega)\left(\omega+\left(\omega^{2}-4\right) S_{m-1}(\omega) S_{m}(\omega)\right)+S_{m}(\omega)=S_{3 m}(\omega)$.

We now simplify the polynomial $\varphi$ by writing the trace polynomials in terms of these Chebyshev polynomials.

Proposition 3.9 We have

$$
\begin{aligned}
t=\left(x S_{m}(z)-y S_{m-1}(z)\right)\left(y S_{m}(z)-x\right. & \left.S_{m-1}(z)\right) \\
& -z\left(S_{m}^{2}(z)+S_{m-1}^{2}(z)\right)+4 S_{m}(z) S_{m-1}(z) .
\end{aligned}
$$


Proof By definition, $t=P_{w_{k}}$. By applying Lemma 3.7 twice, we have

$$
\begin{aligned}
P_{w_{k}}= & P_{\left(a b^{-1}\right)^{m} a b\left(a^{-1} b\right)^{m}} \\
= & S_{m}^{2}(z) P_{a b}+S_{m-1}^{2}(z) P_{\left(a b^{-1}\right)^{-1} a b\left(a^{-1} b\right)^{-1}} \\
& \quad-S_{m}(z) S_{m-1}(z)\left(P_{\left(a b^{-1}\right)^{-1} a b}+P_{a b\left(a^{-1} b\right)^{-1}}\right) \\
= & S_{m}^{2}(z) P_{a b}+S_{m-1}^{2}(z) P_{b a}-S_{m}(z) S_{m-1}(z)\left(P_{b^{2}}+P_{a^{2}}\right) \\
= & \left(S_{m}^{2}(z)+S_{m-1}^{2}(z)\right)(x y-z)-S_{m}(z) S_{m-1}(z)\left(x^{2}+y^{2}-4\right) .
\end{aligned}
$$

The proposition follows.

Proposition 3.10 The polynomial $\varphi(x, y, z) \in \mathbb{C}^{3}[x, y, z]$ is

$$
\varphi(x, y, z)=\left(x y z+4-x^{2}-y^{2}-z^{2}\right)\left(S_{n}(t) S_{m-1}(z)-S_{n-1}(t) S_{m}(z)\right),
$$

where $t$ is as in Proposition 3.9.

Proof As mentioned above, by [16, Theorem 1] $X(k, l)$ is the zero set of $\varphi(x, y, z)$ and $P_{\overleftarrow{r} a b}=P_{b a r}$. By applying Lemma 3.7 we have

$$
\begin{aligned}
P_{r a b}-P_{b a r}= & P_{w_{k}^{n}\left(a b^{-1}\right)^{m} a b}-P_{b a w_{k}^{n}\left(a b^{-1}\right)^{m}} \\
= & S_{n}(t)\left(P_{\left(a b^{-1}\right)^{m} a b}-P_{b a\left(a b^{-1}\right)^{m}}\right) \\
\quad & -S_{n-1}(t)\left(P_{w_{k}^{-1}\left(a b^{-1}\right)^{m} a b}-P_{b a w_{k}^{-1}\left(a b^{-1}\right)^{m}}\right) \\
= & S_{n}(t)\left(P_{\left(a b^{-1}\right)^{m} a b}-P_{b a\left(a b^{-1}\right)^{m}}\right) \\
& \quad-S_{n-1}(t)\left(P_{\left(a^{-1} b\right)^{m}}-P_{\left.a b\left(a^{-1} b\right)^{m}(b a)^{-1}\right),}\right.
\end{aligned}
$$

where

$$
\begin{aligned}
& P_{\left(a b^{-1}\right)^{m} a b}-P_{b a\left(a b^{-1}\right)^{m}}=S_{m}(z)\left(P_{a b}-P_{b a}\right) \\
& -S_{m-1}(z)\left(P_{\left(a b^{-1}\right)^{-1} a b}-P_{b a\left(a b^{-1}\right)^{-1}}\right) \\
& =-S_{m-1}(z)\left(P_{b^{2}}-P_{b a b a^{-1}}\right) \\
& =S_{m-1}(z)\left(x y z+4-x^{2}-y^{2}-z^{2}\right) \text {, } \\
& P_{\left(a^{-1} b\right)^{m}}-P_{a b\left(a^{-1} b\right)^{m}(b a)^{-1}}=S_{m}(z)\left(P_{1}-P_{a b(b a)^{-1}}\right) \\
& -S_{m-1}(z)\left(P_{\left(a^{-1} b\right)^{-1}}-P_{a b\left(a^{-1} b\right)^{-1}(b a)^{-1}}\right) \\
& =S_{m}(z)\left(x y z+4-x^{2}-y^{2}-z^{2}\right) \text {. }
\end{aligned}
$$

Hence

$$
P_{r a b}-P_{b a r}=\left(x y z+4-x^{2}-y^{2}-z^{2}\right)\left(S_{n}(t) S_{m-1}(z)-S_{n-1}(t) S_{m}(z)\right) .
$$


The character variety $X(k, l)$ is clearly reducible. The set of reducible characters, $X_{\text {red }}(k, l)$, can easily be determined, as in [1], for example. We have the following, from which Theorem 1.1 follows immediately.

Proposition 3.11 The vanishing set of

$$
x y z+4-x^{2}-y^{2}-z^{2}
$$

in $\mathbb{C}^{3}(x, y, z)$ is the set of characters of reducible representations $\pi_{1}(k, l) \rightarrow \operatorname{SL}_{2}(\mathbb{C})$. A natural model for the algebraic set $X_{\mathrm{irr}}(k, l)$ is the vanishing set of

$$
S_{n}(t) S_{m-1}(z)-S_{n-1}(t) S_{m}(z)
$$

in $\mathbb{C}^{3}(x, y, z)$, where $t$ is as in Proposition 3.9.

In light of this, we wish to understand the vanishing set of the difference $S_{n}(t) S_{m-1}(z)-$ $S_{n-1}(t) S_{m}(z)$. The equation $S_{n}(t) S_{m-1}(z)=S_{n-1}(t) S_{m}(z)$ can be written as

$$
\frac{S_{n}(t)}{S_{n-1}(t)}=\frac{S_{m}(z)}{S_{m-1}(z)}
$$

when $S_{n-1}(t) S_{m-1}(z) \neq 0$, so we can think of it as lying in a product of projective lines. We will make use of this approach when proving smoothness and irreducibility.

Definition 3.12 Let $V(k, l)$ be the vanishing set of $S_{n}(t) S_{m-1}(z)-S_{n-1}(t) S_{m}(z)$ in $\mathbb{C}^{3}(x, y, z)$.

By Proposition 3.11 the components of $X(k, l)$ containing characters of irreducible representations, those included in $X_{\text {irr }}(k, l)$, are contained in $V(k, l)$ and $V(k, l)$ is a natural model for this set.

\section{The structure of $V(k, l)$}

The set $V(k, l)$ is the closure of the set of characters of irreducible representations. The equation $S_{n}(t) S_{m-1}(z)-S_{n-1}(t) S_{m}(z)$ is relatively simple, except that $t$ itself is a function of the natural variables $x, y$, and $z$. Explicitly, by Proposition 3.9,

$$
\begin{aligned}
t=\left(x S_{m}(z)-y S_{m-1}(z)\right)\left(y S_{m}(z)-x\right. & \left.S_{m-1}(z)\right) \\
& -z\left(S_{m}^{2}(z)+S_{m-1}^{2}(z)\right)+4 S_{m}(z) S_{m-1}(z) .
\end{aligned}
$$

We will show that there is a relatively simple model for $X_{\text {irr }}(k, l)$ up to birational equivalence. 
Definition 4.1 Let $u=x S_{m}(z)-y S_{m-1}(z)$ and $v=y S_{m}(z)-x S_{m-1}(z)$.

It follows that

$$
t=u v-z\left(S_{m}^{2}(z)+S_{m-1}^{2}(z)\right)+4 S_{m}(z) S_{m-1}(z) .
$$

By the definitions of $u$ and $v$,

$$
x=\frac{u S_{m}(z)+v S_{m-1}(z)}{S_{m}^{2}(z)-S_{m-1}^{2}(z)} \quad \text { and } \quad y=\frac{v S_{m}(z)+u S_{m-1}(z)}{S_{m}^{2}(z)-S_{m-1}^{2}(z)} .
$$

We will show that this substitution of $u$ and $v$ for $x$ and $y$ corresponds to a birational map, simplifying the definition of $t$. Then we will show that substituting $t$ for $u$ is another birational map, thus eliminating the problem of having nested variables. This has the fortunate consequence that the equation $S_{n}(t) S_{m-1}(z)-S_{n-1}(t) S_{m}(z)$ contains no $u$, so we can conclude that the algebraic set $V(k, l)$ is birational to the product of a curve and $\mathbb{C}$.

Definition 4.2 Let $U(k, l)$ be the vanishing set of

$$
S_{n}(t) S_{m-1}(z)-S_{n-1}(t) S_{m}(z)
$$

in $\mathbb{C}^{3}(u, v, z)$, where

$$
t=u v-z\left(S_{m}^{2}(z)+S_{m-1}^{2}(z)\right)+4 S_{m}(z) S_{m-1}(z) .
$$

Before showing that $V(k, l)$ is birational to $U(k, l)$ we prove a lemma.

Lemma 4.3 On $V(k, l), S_{m}^{2}(z)-S_{m-1}^{2}(z)=0$ only for a set of codimension one.

Proof By definition $S_{j}(z)$ is a Chebyshev polynomial, and by Lemma 3.8 we have that $S_{m}^{2}(z)-S_{m-1}^{2}(z)=S_{2 m}(z)$. Moreover, letting $z=\sigma+\sigma^{-1}$, we can write

$$
S_{2 m}\left(\sigma+\sigma^{-1}\right)=\frac{\sigma^{2 m+1}-\sigma^{-2 m-1}}{\sigma-\sigma^{-1}} .
$$

Therefore, if $S_{m}^{2}(z)-S_{m-1}^{2}(z)=0$ then $\sigma^{2 m+1}-\sigma^{-2 m-1}=0$ and so $\sigma^{4 m+2}=1$. It follows that

$$
\sigma=e^{2 \pi i s /(4 m+2)}=e^{\pi i s /(2 m+1)}
$$

for some $0 \leq s \leq 4 m+2$. When $s=2 r$ is even $(1 \leq r \leq m)$,

$$
z=\sigma+\sigma^{-1}=2 \operatorname{Re}(\sigma)=2 \cos \left(\frac{2 \pi r}{2 m+1}\right)
$$


and $z$ is a root of $S_{m}(z)+S_{m-1}(z)$. When $s=2 r+1$ is odd $(0 \leq r \leq m-1)$,

$$
z=2 \cos \left(\frac{(2 r+1) \pi}{2 m+1}\right)
$$

and $z$ is a root of $S_{m}(z)-S_{m-1}(z)$.

First, we will show that $S_{m}(z)-S_{m-1}(z)=0$ only for a set of dimension one on $V(k, l)$. By Lemma 3.8,

$$
S_{m}^{2}(z)+S_{m-1}^{2}(z)-z S_{m}(z) S_{m-1}(z)=1 .
$$

Since $S_{m}(z)=S_{m-1}(z)$, we obtain $S_{m}^{2}(z)=1 /(2-z)$ and

$$
t=-S_{m}^{2}(z)\left((x-y)^{2}+2 z-4\right)=\frac{1}{z-2}\left((x-y)^{2}+2(z-2)\right)=\frac{(x-y)^{2}}{z-2}+2 .
$$

We conclude that

$$
(x-y)^{2}=(z-2)(t-2) .
$$

On $V(k, l), S_{n}(t) S_{m-1}(z)-S_{n-1}(t) S_{m}(z)=0$. Since $S_{m}(z)=S_{m-1}(z)$ we get

$$
S_{m}(z)\left(S_{n}(t)-S_{n-1}(t)\right)=0 .
$$

Since $z$ is as above, we see that $S_{m}(z) \neq 0$ since $S_{m}^{2}(z)=1 /(2-z)$. Hence $S_{n}(t)-S_{n-1}(t)=0$. It follows that

$$
t=2 \cos \left(\frac{(2 s+1) \pi}{2 n+1}\right)
$$

where $0 \leq s \leq n-1$. We conclude that

$$
(x-y)^{2}=4\left(\cos \left(\frac{(2 r+1) \pi}{2 m+1}\right)-1\right)\left(\cos \left(\frac{(2 s+1) \pi}{2 n+1}\right)-1\right) .
$$

This defines $x-y$ explicitly, and therefore determines a set of dimension one in $V(k, l)$. Since the dimension of $V(k, l)$ is two, this is a codimension-one set.

We complete the proof by showing that $S_{m}(z)+S_{m-1}(z)=0$ only for a set of dimension one on $V(k, l)$. Note that $z=2 \cos \left(\frac{2 \pi r}{2 m+1}\right)$, where $1 \leq r \leq m$. We have

$$
S_{m}^{2}(z)+S_{m-1}^{2}(z)-z S_{m}(z) S_{m-1}(z)=1 .
$$

Since $S_{m}(z)=-S_{m-1}(z)$, we obtain $S_{m}^{2}(z)=1 /(2+z)$ and

$$
t=S_{m}^{2}(z)\left((x+y)^{2}-2 z-4\right)=\frac{1}{2+z}\left((x+y)^{2}-2(z+2)\right)=\frac{(x+y)^{2}}{2+z}-2 .
$$

We conclude that

$$
(x+y)^{2}=(t+2)(z+2) \text {. }
$$


On $V(k, l)$ we have $S_{n}(t) S_{m-1}(z)-S_{n-1}(t) S_{m}(z)=0$, and hence

$$
S_{m}(z)\left(S_{n}(t)+S_{n-1}(t)\right)=0 .
$$

Since $z$ is as above, we conclude that $S_{n}(t)+S_{n-1}(t)=0$. This means $t=2 \cos \left(\frac{2 \pi s}{2 n+1}\right)$ (where $1 \leq s \leq n$ ). Hence

$$
(x+y)^{2}=4\left(\cos \left(\frac{2 \pi r}{2 m+1}\right)+1\right)\left(\cos \left(\frac{2 \pi s}{2 n+1}\right)+1\right) .
$$

This defines $x+y$ explicitly, and therefore determines a set of dimension one in $V(k, l)$. Since the dimension of $V(k, l)$ is two, this is a codimension-one set.

The next result now easily follows.

Proposition 4.4 The set $V(k, l) \subset \mathbb{C}^{3}(x, y, z)$ is birational to $U(k, l) \subset \mathbb{C}^{3}(u, v, z)$.

Proof As discussed above, the substitution defines a rational map between $V(k, l)$ and $U(k, l)$, namely

$$
(x, y, z) \mapsto\left(\frac{x S_{m}(z)+y S_{m-1}(z)}{S_{m}^{2}(z)-S_{m-1}^{2}(z)}, \frac{y S_{m}(z)+x S_{m-1}(z)}{S_{m}^{2}(z)-S_{m-1}^{2}(z)}, z\right),
$$

with inverse

$$
(u, v, z) \mapsto\left(u S_{m}(z)-v S_{m-1}(z), v S_{m}(z)-u S_{m-1}(z), z\right) .
$$

It suffices to see that $S_{m}^{2}(z)-S_{m-1}^{2}(z)=0$ only for a set of codimension one on $V(k, l)$, which follows from Lemma 4.3.

We now wish to perform one more birational transformation.

Definition 4.5 Let $W(k, l)$ be the vanishing set of

$$
S_{n}(t) S_{m-1}(z)-S_{n-1}(t) S_{m}(z)
$$

in $\mathbb{C}^{3}(t, v, z)$.

For each odd integer $l$, let $W_{0}(l, l)$ denote the component of $W(l, l)$ given by $t=z$ and if $|l|>3$ let $W_{1}(l, l)$ denote the projective closure of the scheme-theoretic complement of $W_{0}(l, l)$ in $W(l, l)$.

First, we prove a lemma.

Lemma 4.6 On $U(k, l), v=0$ only for a set of dimension zero. 
Proof If $v=0$ then since

$$
t=u v-z\left(S_{m}^{2}(z)+S_{m-1}^{2}(z)\right)+4 S_{m}(z) S_{m-1}(z)
$$

we conclude that

$$
t=-z\left(S_{m}^{2}(z)+S_{m-1}^{2}(z)\right)+4 S_{m}(z) S_{m-1}(z) .
$$

The defining polynomial for $U(k, l)$ is $S_{n}(t) S_{m-1}(z)-S_{n-1}(t) S_{m}(z)$. Upon substituting the above polynomial in $\mathbb{Z}[z]$ for $t$ we see that this defining polynomial can be expressed as a polynomial in $\mathbb{Z}[z]$. As a result, this has a finite number of roots. For each of these $z$ values, there is one associated $t$, and hence we have a finite number of points on $U(k, l)$ where $v=0$.

Now we are prepared to show the following.

Proposition 4.7 The set $U(k, l) \subset \mathbb{C}^{3}(u, v, z)$ is birational to $W(k, l) \subset \mathbb{C}^{3}(t, v, z)$.

Proof Since $t$ is linear in $u$, we define the rational map from $\mathbb{C}^{3}(u, v, z)$ to $\mathbb{C}^{3}(t, v, z)$ by this replacement. That is, define the rational map

$$
(u, v, z) \mapsto\left(\frac{\left(u+z\left(S_{m}^{2}(z)+S_{m-1}^{2}(z)\right)-4 S_{m}(z) S_{m-1}(z)\right)}{v}, v, z\right)
$$

which has rational inverse

$$
(t, v, z) \mapsto\left(t v-z\left(S_{m}^{2}(z)+S_{m-1}^{2}(z)\right)+4 S_{m}(z) S_{m-1}(z), v, z\right) .
$$

The result now follows from Lemma 4.6.

Definition 4.8 Let $C(k, l)$ be the curve given by the vanishing set of

$$
S_{n}(t) S_{m-1}(z)-S_{n-1}(t) S_{m}(z)
$$

in $\mathbb{C}^{2}(t, z)$. For each odd integer $l$, let $C_{0}(l, l)$ denote the component of $C(l, l)$ given by $t=z$ and if $|l|>3$ let $C_{1}(l, l)$ denote the projective closure of the scheme-theoretic complement of $C_{0}(l, l)$ in $C(l, l)$.

With this definition, the surface $W(k, l)$ is a product of the curve $C(k, l)$ and $\mathbb{C}$. We have shown that $V(k, l)$ is birational to $W(k, l)$, which is equivalent to the following, proving the first portion of Theorem 1.2.

Theorem 4.9 The algebraic set $X_{\text {irr }}(k, l)$ is birational to $W(k, l)$, which is, in turn, isomorphic to $C(k, l) \times \mathbb{C}$. 


\section{Smoothness and irreducibility of $W(k, l)$}

We will show that if $k \neq l$ then $W(k, l)$ is smooth and irreducible, and if $k=l$ then $W(l, l)$ has two irreducible components. Since $W(k, l)$ is the product of $C(k, l)$ and $\mathbb{C}$, we will focus on the curve $C(k, l)$. Our proof is similar to [8], but with small modifications. Recall that $k=2 m+1$ and $l=2 n+1$. The equation $S_{n}(t) S_{m-1}(z)=$ $S_{n-1}(t) S_{m}(z)$ can be written as

$$
\frac{S_{n}(t)}{S_{n-1}(t)}=\frac{S_{m}(z)}{S_{m-1}(z)}
$$

when $S_{n-1}(t) S_{m-1}(z) \neq 0$.

Definition 5.1 Let

$$
h_{j}=S_{j} / S_{j-1}, \quad \Delta_{j}=S_{j}^{\prime} S_{j-1}-S_{j} S_{j-1}^{\prime} \quad \text { and } \quad H_{n}=S_{j}^{\prime \prime} S_{j-1}-S_{j-1}^{\prime \prime} S_{j} .
$$

We can rewrite the defining equation for $W(k, l)$ as $h_{n}(t)=h_{m}(z)$, and with this notation the derivative is $h_{j}^{\prime}=\Delta_{j} / S_{j-1}^{2}$.

The following lemma can be verified by using Lemma 3.6.

Lemma 5.2 We have

(a) $\left(\omega^{2}-4\right) \Delta_{j}(\omega)=S_{2 j}(\omega)-(2 j+1)$,

(b) $\left(\omega^{2}-4\right)^{2} H_{j}(\omega)=(2 j-2) \omega S_{2 j}(\omega)-(4 j+2) S_{2 j-1}(\omega)+(4 j+2) \omega$.

We will need the following lemma (see [8, Lemma 2.6]) to connect smoothness and irreducibility.

Lemma 5.3 Let $C \subset \mathbb{P}^{1} \times \mathbb{P}^{1}$ be a smooth projective curve of bidegree $(a, b)$ with $a, b>0$. Then $C$ is irreducible and its genus is $(a-1)(b-1)$.

The proof of smoothness will follow from comparing valuations at potential critical points. We begin with a few lemmas. In the case that $m n<0$ we use the following lemma.

Lemma 5.4 Let $\omega \in \mathbb{C}$ be a root of $\Delta_{n}$. If $n>0$ then $\left|h_{n}(\omega)\right|>1$, and if $n<0$ then $\left|h_{n}(\omega)\right|<1$. 
Proof Suppose that $\Delta_{n}(\omega)=0$. By Lemma 5.2, $S_{2 n}(\omega)=2 n+1$. We have $S_{n-1}(\omega) \neq 0$ (otherwise

$$
S_{n}(\omega) S_{n-1}^{\prime}(\omega)=S_{n}^{\prime}(\omega) S_{n-1}(\omega)-\Delta_{n}(\omega)=0,
$$

which cannot occur, since $S_{n-1}$ is separable and relatively prime to $S_{n}$ in $\mathbb{C}[\omega]$ ). Hence $h_{n}(\omega)=S_{n}(\omega) / S_{n-1}(\omega)$ is well-defined. Write $\omega=\sigma+\sigma^{-1}$. We have $S_{2 n}(\omega)=2 n+1$, ie

$$
\sigma^{2 n+1}-\sigma^{-(2 n+1)}=(2 n+1)\left(\sigma-\sigma^{-1}\right) .
$$

Assume $n>0$. Then $\sigma^{2 n+1}-\sigma^{-(2 n+1)}$ and $\sigma-\sigma^{-1}$ are in the same half-plane. It follows that $\sigma^{2 n+1}-\bar{\sigma}^{2 n+1}$ and $\sigma-\bar{\sigma}$ are in the same half-plane. Since both these values are purely imaginary, we conclude $\left(\sigma^{2 n+1}-\bar{\sigma}^{2 n+1}\right)(\sigma-\bar{\sigma}) \leq 0$, with equality if and only if $\sigma^{2 n+1}$ is real.

Let $\alpha=\sigma \bar{\sigma}=|\sigma|^{2}>0$. We have

$$
\begin{aligned}
\mid \sigma^{n+1}- & \left.\sigma^{-(n+1)}\right|^{2}-\left|\sigma^{n}-\sigma^{-n}\right|^{2} \\
& =\left(\sigma^{n+1}-\sigma^{-(n+1)}\right)\left(\bar{\sigma}^{n+1}-\bar{\sigma}^{-(n+1)}\right)-\left(\sigma^{n}-\sigma^{-n}\right)\left(\bar{\sigma}^{n}-\bar{\sigma}^{-n}\right) \\
& =\left(\alpha^{n+1}+\alpha^{-(n+1)}-\left(\alpha^{n}+\alpha^{-n}\right)\right)-\left(\sigma^{2 n+1}-\bar{\sigma}^{2 n+1}\right)(\sigma-\bar{\sigma}) / \sigma^{n+1} \bar{\sigma}^{n+1} \\
& =(\alpha-1)\left(\alpha^{2 n+1}-1\right) / \alpha^{n+1}-\left(\sigma^{2 n+1}-\bar{\sigma}^{2 n+1}\right)(\sigma-\bar{\sigma}) / \alpha^{n+1} \geq 0 .
\end{aligned}
$$

Equality holds if and only if $|\sigma|^{2}=\alpha=1$ and $\sigma^{2 n+1}$ is real, so if and only if $\sigma^{2 n+1}= \pm 1$. If $\sigma^{2 n+1}= \pm 1$, the equation $\sigma^{2 n+1}-\sigma^{-(2 n+1)}=(2 n+1)\left(\sigma-\sigma^{-1}\right)$ implies that $\sigma=\sigma^{-1}$, so $\sigma= \pm 1$ and $\omega= \pm 2$. If $\omega= \pm 2$ then

$$
\left|h_{n}(\omega)\right|=\left|S_{n}(\omega) / S_{n-1}(\omega)\right|=(n+1) / n>1 .
$$

The proof for $n<0$ is similar. In that case $\sigma^{2 n+1}-\bar{\sigma}^{2 n+1}$ and $\sigma-\bar{\sigma}$ are in opposite half-planes and $(\alpha-1)\left(\alpha^{2 n+1}-1\right) \leq 0$.

In the remaining case $(m n>0)$ we can use non-archimedean places instead of complex absolute values. For any root $\omega$ of $\Delta_{n}$, we have $S_{2 n}(\omega)=2 n+1$. It follows that

$$
h_{n}^{2}(\omega)-1=\left(\frac{S_{n}(\omega)}{S_{n-1}(\omega)}\right)^{2}-1=\frac{S_{2 n}(\omega)}{S_{n-1}^{2}(\omega)}=\frac{2 n+1}{S_{n-1}^{2}(\omega)} .
$$

Lemma 5.5 For any field $\mathbb{F}$ with characteristic not dividing $2 n$, the polynomial $S_{n-1}$ is separable over $\mathbb{F}$ and we have $\left(\Delta_{n}, S_{n-1}\right)=(1)$ in $\mathbb{F}[\omega]$.

Proof We have $\left(\sigma^{n+1}-\sigma^{n-1}\right) S_{n-1}=\sigma^{2 n}-1$, and the reduction of this polynomial to $\mathbb{F}$ is separable. It follows that $S_{n-1}$ is separable over $\mathbb{F}$, ie $\left(S_{n-1}, S_{n-1}^{\prime}\right)=(1)$. Since $\Delta_{n}=S_{n}^{\prime} S_{n-1}-S_{n} S_{n-1}^{\prime}$, we have $\left(\Delta_{n}, S_{n-1}\right)=\left(S_{n} S_{n-1}^{\prime}, S_{n-1}\right)=(1)$. 
Lemma 5.6 Let $p$ be a prime dividing $2 n+1$. Let $K$ be a number field containing a root $\omega$ of $\Delta_{n}$. Let $v$ be a valuation on $K$ with $v(p)=1$. Then $v\left(S_{n-1}(\omega)\right)=0$.

Proof The polynomial $\Delta_{n}$ is monic, so $\omega$ is an algebraic integer. Let $\mathfrak{p}$ be the prime associated with $v$, and $\mathbb{F}_{\mathfrak{p}}$ be its residue field. Then the characteristic $p$ of $\mathbb{F}_{\mathfrak{p}}$ does not divide $2 n$, so by Lemma 5.5 the reduction of $S_{n-1}(\omega)$ to $\mathbb{F}_{\mathfrak{p}}$ is not 0 . This implies $v\left(S_{n-1}(\omega)\right)=0$.

We now address smoothness.

Proposition 5.7 Let $k$ and $l$ be any odd integers with $k \neq l$. Then $C(k, l)$ is smooth over $\mathbb{Q}$.

Proof Suppose $P=\left(t_{0}, z_{0}\right)$ is a singular point on the affine part of $C(k, l)$. Then $S_{n-1}\left(t_{0}\right) \neq 0$ and $S_{m-1}\left(z_{0}\right) \neq 0$. (If $S_{n-1}\left(t_{0}\right)=0$ then $S_{m-1}\left(z_{0}\right)=0$. Since $P$ is a singular point, we also have $S_{n-1}^{\prime}\left(t_{0}\right)=0$ and $S_{m-1}^{\prime}\left(z_{0}\right)=0$. This is impossible since $S_{j}$ is separable.) Then $C(k, l)$ can be given around $P$ by $h_{n}(t)=h_{m}(z)$. The fact that $P$ is a singular point is then equivalent to the fact that $t_{0}$ and $z_{0}$ are critical points for $h_{n}$ and $h_{m}$, respectively. (We have $\Delta_{n}\left(t_{0}\right)=\Delta_{m}\left(z_{0}\right)=0$, ie $h_{n}^{\prime}\left(t_{0}\right)=h_{m}^{\prime}\left(z_{0}\right)=0$.)

First, consider the case when $k l<0$. The points at infinity are smooth by $[8$, Lemma 5.6]. The proposition follows from Lemma 5.4. That is, the values of $h_{k}$ at its critical points are all different from each other, and they are also different from the values of $h_{l}$ at all its critical points when $k \neq l$.

Now, assume that $k l>0$ but $k \neq l$. Assume $P\left(t_{0}, z_{0}\right)$ is a singular point over $\overline{\mathbb{Q}}$ of the standard affine part of $C(k, l)$. Let $K$ be the number field $\mathbb{Q}\left(t_{0}, z_{0}\right)$. We have $\Delta_{n}\left(t_{0}\right)=\Delta_{m}\left(z_{0}\right)=0$ and $C(k, l)$ is given around $P$ by $h_{n}\left(t_{0}\right)=h_{m}\left(z_{0}\right)$. It follows that $h_{n}^{2}\left(t_{0}\right)-1=h_{m}^{2}\left(z_{0}\right)-1$, ie

$$
\frac{2 n+1}{S_{n-1}^{2}\left(t_{0}\right)}=\frac{2 m+1}{S_{m-1}^{2}\left(z_{0}\right)}
$$

Let $p$ be any prime such that $v_{p}(2 n+1) \neq v_{p}(2 m+1)$. By symmetry we may assume $v_{p}(2 n+1)>v_{p}(2 m+1)$. Let $\mathfrak{p}$ be any prime of $K$ above $p$, and let $v$ be the valuation on $K$ associated to $p$, normalized so that $v$ restricts to $v_{p}$ on $\mathbb{Q}$. By Lemma 5.6, we have

$$
v\left(\frac{2 n+1}{S_{n-1}^{2}\left(t_{0}\right)}\right)=v(2 n+1)>v(2 m+1) \geq v\left(\frac{2 m+1}{S_{m-1}^{2}\left(z_{0}\right)}\right) .
$$

This contradicts the equality $(*)$, and we conclude that no singular point $P$ exists on the affine part. By $[8$, Lemma 5.6] there are no singular points at infinity. 
Proposition 5.8 Let $l$ be any odd integer. Then the curve $C_{1}(l, l)$ is smooth over $\mathbb{Q}$.

Proof Let $F=S_{n}(t) S_{n-1}(z)-S_{n-1}(t) S_{n}(z)$ and $G=F /(z-t)$. Then $C_{1}(l, l)$ is defined by $G(t, z)=0$. Any singular point of $C_{1}(l, l)$ is also a singular point of $C(l, l)$. By [8, Lemma 5.6] we find that $C(l, l)$ is smooth at all points at infinity, so $C_{1}(l, l)$ is as well. Assume that $P=\left(t_{0}, z_{0}\right)$ is a singular point of the standard affine part of $C_{1}(l, l)$. Then $P$ is also a singular point of $C(l, l)$. Note that $\Delta_{n}\left(t_{0}\right)=0$ and $\Delta_{n}\left(z_{0}\right)=0$, and we may rewrite $F(P)=0$ as $h_{n}\left(t_{0}\right)=h_{n}\left(z_{0}\right)$. Recall $\left(\omega^{2}-4\right) \Delta_{n}(\omega)=S_{2 n}(\omega)-(2 n+1)$.

Since $S_{n}^{2}(\omega)-\omega S_{n}(\omega) S_{n-1}(\omega)+S_{n-1}^{2}(\omega)=1$ and $S_{n}^{2}(\omega)-S_{n-1}^{2}(\omega)=S_{2 n}(\omega)$, we have

$$
h_{n}(\omega)+h_{n}^{-1}(\omega)=\omega+\frac{1}{S_{n}(\omega) S_{n-1}(\omega)}
$$

and

$$
h_{n}(\omega)-h_{n}^{-1}(\omega)=\frac{S_{2 n}(\omega)}{S_{n}(\omega) S_{n-1}(\omega)} .
$$

Since $h_{n}\left(t_{0}\right)=h_{n}\left(z_{0}\right)$ and $S_{2 n}\left(t_{0}\right)=S_{2 n}\left(z_{0}\right)=2 n+1$, we conclude that $t_{0}=z_{0}$.

Recall that $H_{n}=S_{n}^{\prime \prime} S_{n-1}-S_{n} S_{n-1}^{\prime \prime}$. By l'Hôpital's rule, we have

$$
\begin{aligned}
-H_{n}\left(t_{0}\right) & =F_{z z}\left(t_{0}, t_{0}\right) \\
& =\lim _{z \rightarrow t_{0}} \frac{F_{z}\left(t_{0}, z\right)}{z-t_{0}}=2 \lim _{z \rightarrow t_{0}} \frac{F\left(t_{0}, z\right)}{\left(z-t_{0}\right)^{2}}=2 \lim _{z \rightarrow t_{0}} \frac{G\left(t_{0}, z\right)}{z-t_{0}} \\
& =2 G_{z}\left(t_{0}, t_{0}\right) .
\end{aligned}
$$

The fact that $C_{1}(l, l)$ is singular at $P=\left(t_{0}, t_{0}\right)$ implies that $0=G_{z}(P)=-\frac{1}{2} H_{n}\left(t_{0}\right)$. Hence, by Lemma 5.2 we have

$$
(2 n-2) t_{0} S_{2 n}\left(t_{0}\right)-(4 n+2) S_{2 n-1}\left(t_{0}\right)+(4 n+2) t_{0}=\left(t_{0}^{2}-4\right)^{2} H_{n}\left(t_{0}\right)=0 .
$$

Since $S_{2 n}\left(t_{0}\right)=2 n+1$, we obtain $S_{2 n-1}\left(t_{0}\right)=n t_{0}$. Since

$$
S_{2 n}^{2}\left(t_{0}\right)-t_{0} S_{2 n}\left(t_{0}\right) S_{2 n-1}\left(t_{0}\right)+S_{2 n-1}^{2}\left(t_{0}\right)=1,
$$

we conclude that $t_{0}= \pm 2$. This is a contradiction, since $\Delta_{n}( \pm 2) \neq 0$ by direct calculation. We are done.

Proposition 5.9 The algebraic set $C(k, l)$ is smooth and has one irreducible component if $k \neq l$. The curve $C(3,3)=C(-3,-3)$ is given by $t=z$. If $k=l$ and $|l|>3$ then $C(k, l)$ has two irreducible components, $C_{0}(l, l)$ and $C_{1}(l, l)$. Both $C_{0}(l, l)$ and $C_{1}(l, l)$ are smooth. 
Proof By Lemma 5.3 it suffices to show that $C(k, l)$ is smooth. If $k \neq l$, then $C(k, l)$ is smooth by Proposition 5.7. If $k=l$ then $C_{1}(l, l)$ is smooth by Proposition 5.8. The proposition follows since $C_{0}(l, l)$ is given by $t=z$ and is smooth.

We have shown that if $k \neq l$ then $X_{\text {irr }}(k, l)$ is a single irreducible component. When $k=l$ and $|l|>3$, we have shown that $X_{\text {irr }}(k, l)$ comprises two irreducible components, and we now identify the canonical component.

Lemma 5.10 If $k \neq l$ then $X_{0}(k, l)$ is birational to $C_{0}(k, l) \times \mathbb{C}$. The curve $C(3,3)=$ $C(-3,-3)$ is given by $t=z$ and $X_{0}(3,3)$ is birational to $C(3,3) \times \mathbb{C}$. If $k=l$ and $|l|>3$ then $X_{0}(l, l)$ is birational to $C_{0}(l, l) \times \mathbb{C}$ and there is one more irreducible component of $X_{\mathrm{irr}}(l, l)$, birational to $C_{1}(l, l) \times \mathbb{C}$.

Proof By Theorem 4.9, $X_{\mathrm{irr}}(k, l)$ is birational to $C(k, l) \times \mathbb{C}$. Proposition 5.9 shows that $X_{0}(k, l)=X_{\text {irr }}(k, l)$ when $k \neq l$.

By the definition of $C_{0}(l, l)$ it suffices to show that $t=z$ corresponds to the canonical component. By construction, $z=\chi_{\rho}\left(a b^{-1}\right)$ corresponds to the loop $\alpha$ pictured in Figure 2. Moreover, $t=\chi_{\rho}\left(w_{k}\right)$ corresponds to the loop $\beta$ pictured in the figure. When $k=l$ the symmetry induced by flipping the four-plat upside down swaps these loops. On the level of the character variety this symmetry induces the identity $t=z$. (The symmetry acts trivially on $x=\chi_{\rho}(a)$ and $y=\chi_{\rho}(b)$.) For any discrete faithful representation, $t=z$ must hold on the level of characters since the loops corresponding to $z$ and $t$ must have the same length (since they are swapped by the symmetry). The symmetry sends each meridian to a loop freely homotopic to itself, with the reverse orientation, and does the same for each longitude. Therefore, the symmetry induces a symmetry on any Dehn filling of the link. We conclude that $t=z$ must be satisfied by all Dehn fillings as well. By work of Thurston [14], all but finitely many Dehn fillings of one cusp of the link are on canonical components, and so are dense in $X_{0}(k, l)$. (See [9] and also [8, Section 2.3]) The fact that there are exactly two irreducible components in this case follows from Proposition 5.9.

We summarize this section in the following theorem.

Theorem 1.2 Let $k=2 m+1$ and $l=2 n+1$. The algebraic set $X_{\text {irr }}(k, l)$ is birational to $C(k, l) \times \mathbb{C}$, where the curve $C(k, l) \subset \mathbb{C}^{2}(t, z)$ is given by

$$
C(k, l)=\left\{S_{n}(t) S_{m-1}(z)-S_{n-1}(t) S_{m}(z)=0\right\} .
$$

If $k \neq l$ then $C(k, l)$ is smooth and irreducible as considered in $\mathbb{P}^{1}(t) \times \mathbb{P}^{1}(z)$, and $X_{0}(k, l)=X_{\text {irr }}(k, l)$ is birational to $C(k, l) \times \mathbb{C}$. 

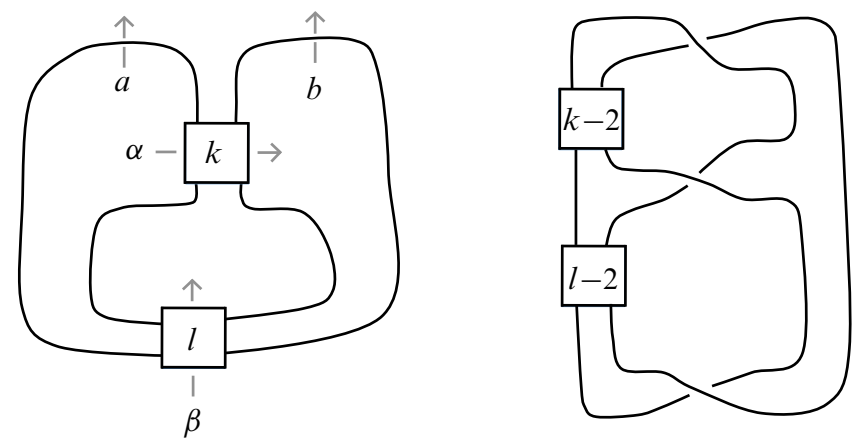

Figure 2: Meridian loops on double twist links and the four-plat presentation

The curve $C(3,3)=C(-3,-3)$ is given by $t=z$. If $k=l$ and $|l|>3$ then $C(l, l)$ is the union of exactly two components: $C_{0}(l, l)$, given by $t=z$, and $C_{1}(l, l)$, the scheme-theoretic complement of $C_{0}(l, l)$ in $C(l, l)$. Both are smooth and irreducible as considered in $\mathbb{P}^{1}(t) \times \mathbb{P}^{1}(z)$. The algebraic set $X_{\text {irr }}(k, l)$ is given by the union $X_{0}(l, l) \cup X_{1}(l, l)$, where $X_{0}(l, l)$ is birational to $C_{0}(l, l) \times \mathbb{C}$ and $X_{1}(l, l)$ is birational to $C_{1}(l, l) \times \mathbb{C}$.

We conclude this section with a few remarks about symmetries. The proof of Lemma 5.10 relied on analysis of the symmetry which flips the four-plat upside down. For all $k$ and $l$, the link complement $S^{3}-J(k, l)$ has a non-trivial symmetry group. In the case when $k \neq l$ this is generated by two involutions. The first is the flip about a vertical axis through the $k$ half-twists. (In the left projection in Figure 2 this axis is the vertical axis through the middle of the diagram.) The second symmetry is the analogous symmetry through an axis through the $l$ half-twists. (In the left projection in Figure 2 this axis is a circle through the middle of the $l$ half-twists which goes horizontally through the $k$ box.)

These symmetries both take the loop corresponding to $a$ to a loop freely homotopic to the loop corresponding to $b^{-1}$, and fix the free homotopy class of the un-oriented loop corresponding to $a b^{-1}$. Since they are involutions, the effect on the character variety is that $x=\chi_{\rho}(a)$ is sent to $y=\chi_{\rho}(b)=\chi_{\rho}\left(b^{-1}\right)$ and $z=\chi_{\rho}\left(a b^{-1}\right)$ is fixed. By definition, $t=\chi_{\rho}\left(w_{k}\right)$ is given by

$$
\begin{aligned}
& \left(x S_{m}(z)-y S_{m-1}(z)\right)\left(y S_{m}(z)-x S_{m-1}(z)\right) \\
& -z\left(S_{m}^{2}(z)+S_{m-1}^{2}(z)\right)+4 S_{m}(z) S_{m-1}(z) .
\end{aligned}
$$

We conclude that these symmetries fix $t$. Therefore, the induced action of the symmetry group on $\mathbb{C}^{3}[x, y, z]$ when $k \neq l$ is given by $(x, y, z) \mapsto(y, x, z)$. (This is the action of an index-two subgroup when $k=l$.) 
Recall that

$$
\varphi(x, y, z)=\left(x y z+4-x^{2}-y^{2}-z^{2}\right)\left(S_{n}(t) S_{m-1}(z)-S_{n-1}(t) S_{m}(z)\right) .
$$

The abelian component of the character variety is given by $\left(x y z+4-x^{2}-y^{2}-z^{2}\right)$ and is preserved by this action. As there are points on this component where $x \neq y$, we conclude that the action preserves this component set-wise but not point-wise. (For example, the point $(2,-2,2)$ is sent to $(-2,2,2)$.) The set of irreducible representations is given by $\left(S_{n}(t) S_{m-1}(z)-S_{n-1}(t) S_{m}(z)\right)$. Since $t$ and $z$ are fixed, this component (or in the case when $k=l$, these two components) is fixed by these symmetries. When $k \neq l$, since $x \neq y$ for infinitely many representations on this component, we conclude that the action preserves this component set-wise but not point-wise. Similarly, when $k=l$ these symmetries preserve both $z=t$ and the other component set-wise but not point-wise.

We conclude that even the non-geometric representations algebraically preserve this symmetry. However, when $k=l$ the additional symmetry fixes the un-oriented free homotopy class of loops corresponding to $a$ and similarly for $b$, but takes the unoriented loop corresponding to $a b^{-1}$ to a loop freely homotopic to one corresponding to $w_{k}$. This is not freely homotopic to $\left(a b^{-1}\right)^{ \pm 1}$. It is this that induces the factoring of the defining equation, $\varphi$. In this case, when $|l|>3$ there is a component which corresponds to necessarily non-geometric representations which do not algebraically preserve this symmetry.

\section{Further invariants}

We have shown in Theorem 1.2 that, when $k \neq l, X_{0}(k, l)$ is birational to $C_{0}(k, l) \times \mathbb{C}$, and that $C_{0}(k, l)$ is smooth and irreducible in $\mathbb{P}^{1} \times \mathbb{P}^{1}$. We have also shown that $X_{\text {irr }}(l, l)$ is birational to the union of $C_{0}(l, l) \times \mathbb{C}$ and $C_{1}(l, l) \times \mathbb{C}$. We now compute the genus of these curves, and the degree of irrationality of $X_{0}(k, l)$ and $X_{1}(l, l)$.

Lemma 6.1 When $k \neq l$ the bidegree of $C(k, l)$ is $\left(\left\lfloor\frac{\lfloor k \mid}{2}\right\rfloor,\left\lfloor\frac{|l|}{2}\right\rfloor\right)$. The bidegree of $C_{1}(l, l)$ is $\left(\left\lfloor\frac{|l|}{2}\right\rfloor-1,\left\lfloor\frac{|l|}{2}\right\rfloor-1\right)$.

Proof By Lemma 3.6, $S_{-1}=0$ and the degree of $S_{j}$ is $j$ when $j>0$ and $-j-2$ when $j<-1$. Therefore, the bidegree of $C(k, l)$ is $(a, b)$, where $a=n$ if $n>0$ and $a=-n-1$ if $n<-1$, and $b=m$ if $m>0$ and $b=-m-1$ if $m<-1$. This is equivalent to $a=\left\lfloor\frac{|k|}{2}\right\rfloor$ and $b=\left\lfloor\frac{|l|}{2}\right\rfloor$. The computation for $C_{1}(l, l)$ follows from this using the definition of $C_{1}(l, l)$. 
Theorem 1.3 Let $|k|,|l|>1$. When $k \neq l$ the genus of $C(k, l)$ is

$$
\left(\left\lfloor\frac{|k|}{2}\right\rfloor-1\right)\left(\left\lfloor\frac{|l|}{2}\right\rfloor-1\right) .
$$

The genus of $C_{0}(l, l)$ is zero, and for $|l|>3$ the genus of $C_{1}(l, l)$ is $\left(\left\lfloor\frac{|l|}{2}\right\rfloor-2\right)^{2}$.

Proof The result follows from the following, by Lemma 6.1. If $C$ is a smooth projective curve in $\mathbb{P}^{1} \times \mathbb{P}^{1}$ of bidegree $(a, b)$ then the genus is $(a-1, b-1)$ (see [8]).

Definition 6.2 Let $X$ be an irreducible (affine or projective) complex variety of dimension $n$. The degree of irrationality of $X, \gamma(X)$, is the minimal degree of any rational map from $X$ to a dense subset of $\mathbb{C}^{n}$. When $X$ is a curve, this is also called the gonality of $X$.

The gonality, in its relation to character varieties and Dehn filling, is discussed at length in [11]. Moreover, the gonality of the components of the $\mathrm{SL}_{2}(\mathbb{C})$ and $\mathrm{PSL}_{2}(\mathbb{C})$ character varieties are computed (Theorem 9.2, Theorem 9.4). We now compute the degree of irrationality of our sets.

Theorem 1.4 The degree of irrationality of $X_{0}(k, l)$ is $\min \left\{\left\lfloor\frac{|k|}{2}\right\rfloor,\left\lfloor\frac{|l|}{2}\right\rfloor\right\}$ when $k \neq l$. The degree of irrationality of $X_{0}(l, l)$ is 1 , and the degree of irrationality of $X_{1}(l, l)$ is $\left\lfloor\frac{|l|}{2}\right\rfloor-1$.

Proof The degree of irrationality of a surface of the form $C \times \mathbb{C}$ is equal to the gonality of $C$; see [18, Proposition 1] and [15]. (If $C$ is a non-singular projective curve then $C \times \mathbb{C}$ is a non-singular projective surface since the fibers have genus zero.) Following [11, Lemma 9.1] if $C$ is a smooth irreducible curve in $\mathbb{P}^{1} \times \mathbb{P}^{1}$ of bidegree $(a, b)$ with $a b \neq 0$ then the gonality of $C$ is $\min \{a, b\}$. The result now follows from Lemma 6.1.

\section{Desingularization of $X_{0}(3,2 m+1)$}

The simplest subfamily of the hyperbolic two-component double twist links is when $k=3$ (so $n=1$ ). This family includes the Whitehead link $5_{1}^{2}=(8 / 3)$ which is $J(3,3)$, and $6_{2}^{2}=(10 / 3)$ which is $J(3,-3)$. In this section we first determine the singular points of the natural model of $X_{0}(3, l)$, where $l=2 m+1$ in Proposition 7.5. In Proposition 7.7 we determine the degenerate fibers of the map $\phi: S \rightarrow \mathbb{P}^{1},(x: y: u, z: w) \mapsto(z: w)$. We then show in Theorem 1.5 that the desingularization of the natural model for $X_{0}(3,2 m+1)$ is a series of blowups of $\mathbb{P}^{1} \times \mathbb{P}^{1}$. 
By Theorem 1.2, $X_{0}(3, l)=X_{\text {irr }}(3, l)$ is birational to $C(3, l) \times \mathbb{C}$, where $C(3, l)$ is given by $t S_{m-1}(z)=S_{m}(z)$ in $\mathbb{C}^{2}(t, z)$. Since this defining polynomial is linear in $t$ we conclude that $C(3, l)$ is itself birational to $\mathbb{C}$ and $X_{0}(3, l)$ is indeed birational to $\mathbb{C}^{2}$. The Whitehead link, $J(-3,-3)=J(3,3)$ is a degenerate case of the $J(3, l)$ links, where $X_{0}(3,3)=X_{\text {irr }}(3,3)$ is given by $t=z$ up to birational equivalence.

We begin by homogenizing the defining polynomial for $X_{0}(3, l)$, where $l=2 m+1$. Recall that

$$
\begin{aligned}
t=\left(x S_{m}(z)-y S_{m-1}(z)\right)\left(y S_{m}(z)-x\right. & \left.S_{m-1}(z)\right) \\
& -z\left(S_{m}^{2}(z)+S_{m-1}^{2}(z)\right)+4 S_{m}(z) S_{m-1}(z) .
\end{aligned}
$$

Since $S_{m}^{2}(z)+S_{m-1}^{2}(z)-z S_{m}(z) S_{m-1}(z)=1$, this simplifies to

$$
t=x y-z+\left(x y z+4-x^{2}-y^{2}-z^{2}\right) S_{m}(z) S_{m-1}(z) .
$$

The defining polynomial for the natural model of $X_{0}(3, l)$ is $t S_{m-1}(z)-S_{m}(z)$ in $\mathbb{C}[x, y, z]$. We now homogenize it.

Definition 7.1 Let $T_{j}=T_{j}(z, w)=w^{j} S_{j}(z / w)$.

The following is a direct consequence of the Chebyshev identity

$$
S_{j}^{2}(\omega)+S_{j-1}^{2}(\omega)-\omega S_{j}(\omega) S_{j-1}(\omega)=1 .
$$

Lemma 7.2 We have

$$
T_{j}^{2}+w^{2} T_{j-1}^{2}-z T_{j} T_{j-1}=w^{2 j}
$$

It is now elementary to determine the homogenous defining polynomial.

Lemma 7.3 The homogenization of the defining polynomial $t S_{m-1}(z)-S_{m}(z)$ in $\mathbb{P}^{2} \times \mathbb{P}^{1}=\{(x: y: u, z: w)\}$ is

$$
\begin{array}{r}
F=\left[\left(x y w-u^{2} z\right) w^{2 m}+\left(x y z w+4 u^{2} w^{2}-x^{2} w^{2}-y^{2} w^{2}-u^{2} z^{2}\right) T_{m} T_{m-1}\right] T_{m-1} \\
-u^{2} w^{2 m} T_{m} .
\end{array}
$$

We now determine the singular points in the projective closure of our natural model in $\mathbb{P}^{2} \times \mathbb{P}^{1}$. To find singular points, we consider solutions $(x: y: u, z: w)$ of $F=F_{x}=F_{y}=F_{u}=F_{z}=F_{w}=0$.

First, we compute these partial derivatives; the results are elementary to verify by direct calculations. 
Lemma 7.4 The first-order partials of $F$ as in Lemma 7.3 are given by the following:

$$
\begin{aligned}
F_{x}=( & \left.y w^{2 m}+(y z-2 x w) T_{m} T_{m-1}\right) w T_{m-1}, \\
F_{y}=( & \left.x w^{2 m}+(x z-2 y w) T_{m} T_{m-1}\right) w T_{m-1}, \\
F_{u}=- & 2 u\left[\left(z w^{2 m}+\left(z^{2}-4 w^{2}\right) T_{m} T_{m-1}\right) T_{m-1}+w^{2 m} T_{m}\right], \\
F_{z}=[ & -u^{2} w^{2 m}+\left(x y w-2 u^{2} z\right) T_{m} T_{m-1} \\
& \left.+\left(x y z w+4 u^{2} w^{2}-x^{2} w^{2}-y^{2} w^{2}-u^{2} z^{2}\right)\left(T_{m} T_{m-1}\right)_{z}\right] T_{m-1} \\
& +\left[\left(x y w-u^{2} z\right) w^{2 m}+\left(x y z w+4 u^{2} w^{2}-x^{2} w^{2}-y^{2} w^{2}-u^{2} z^{2}\right) T_{m} T_{m-1}\right] \\
& \quad \times\left(T_{m-1}\right)_{z}-u^{2} w^{2 m}\left(T_{m}\right)_{z}, \\
F_{w}=[ & (2 m+1) x y w^{2 m}-2 m u^{2} z w^{2 m-1}+\left(x y z+8 u^{2} w-2 x^{2} w-2 y^{2} w\right) T_{m} T_{m-1} \\
& \left.\quad+\left(x y z w+4 u^{2} w^{2}-x^{2} w^{2}-y^{2} w^{2}-u^{2} z^{2}\right)\left(T_{m} T_{m-1}\right)_{w}\right] T_{m-1} \\
& \quad+\left[\left(x y w-u^{2} z\right) w^{2 m}+\left(x y z w+4 u^{2} w^{2}-x^{2} w^{2}-y^{2} w^{2}-u^{2} z^{2}\right) T_{m} T_{m-1}\right] \\
& \quad \times\left(T_{m-1}\right)_{w}-u^{2}\left(2 m w^{2 m-1} T_{m}+w^{2 m}\left(T_{m}\right)_{w}\right) .
\end{aligned}
$$

We can now determine the singular points.

Proposition 7.5 The singular points $(x: y: u, z: w) \in \mathbb{P}^{2} \times \mathbb{P}^{1}$ of $F$ are

- $(1: 0: 0,1: 0),(0: 1: 0,1: 0)$,

- $(1: 0: 0, z: 1),(0: 1: 0, z: 1)$, where $z$ is a root of $S_{m-1}(z)$,

- $(1: 1: 0, z: 1)$, where $z$ is a root of $S_{m}(z)-S_{m-1}(z)$,

- $(1:-1: 0, z: 1)$, where $z$ is a root of $S_{m}(z)+S_{m-1}(z)$.

The number of singularities is $4 m$ if $m \geq 1$, and is $-(2+4 m)$ if $m \leq-2$.

Proof We break the analysis down into cases.

First, we consider the case when $(w: z)=(0: 1)$. We have $F_{x}=F_{y}=0, F=-u^{2}$ and $F_{u}=-2 u$. Hence $u=0$. Now we have $F_{z}=0$ and $F_{w}=x y$. Thus $x y=0$. In this case, there are two singular points, $(1: 0: 0,1: 0)$ and $(0: 1: 0,1: 0)$.

Next, we consider the case when $w=1$. First we assume that $S_{m-1}(z)=0$. Then

$$
F_{x}=F_{y}=0, \quad F=-u^{2} S_{m}(z), \quad F_{u}=-2 u S_{m}(z) .
$$

Since $S_{m}(z) \neq 0$, we have $u=0$. Then $F_{z}=x y S_{m-1}^{\prime}(z)$ and $F_{w}=x y\left(T_{m-1}\right)_{w}$. Since $S_{m-1}^{\prime}(z) \neq 0$, we must have $x y=0$. In this case, the singular points are $(1: 0: 0, z: 1),(0: 1: 0, z: 1)$, where $z$ is a root of $S_{m-1}(z)$. 
Finally, we assume that $w=1$ and $S_{m-1}(z) \neq 0$. We have

$$
\begin{aligned}
& F_{x}=y+(y z-2 x) S_{m}(z) S_{m-1}(z)=y\left(S_{m}^{2}(z)+S_{m-1}^{2}(z)\right)-2 x S_{m}(z) S_{m-1}(z), \\
& F_{y}=x+(x z-2 y) S_{m}(z) S_{m-1}(z)=x\left(S_{m}^{2}(z)+S_{m-1}^{2}(z)\right)-2 y S_{m}(z) S_{m-1}(z) .
\end{aligned}
$$

If $x$ and $y$ are not simultaneously equal to 0 , we must have $S_{m}^{2}(z)-S_{m-1}^{2}(z)=0$.

We first consider the subcase when $x=y=0$, so $(x: y: u)=(0: 0: 1)$. Then, by Lemma 3.8,

$$
F=S_{m-1}(z)\left(-z+\left(4-z^{2}\right) S_{m-1}(z) S_{m}(z)\right)-S_{m}(z)=-S_{3 m}(z) .
$$

Since $S_{3 m}(z)$ is separable in $\mathbb{C}[z]$, there are no singular points in this case.

Therefore, we may assume that $x y \neq 0$ and $S_{m}^{2}(z)-S_{m-1}^{2}(z)=0$. We consider the cases that $S_{m}(z)-S_{m-1}(z)=0$ and $S_{m}(z)+S_{m-1}(z)=0$ separately.

First assume that $S_{m}(z)-S_{m-1}(z)=0$. Then $F_{x}=F_{y}=0$ is equivalent to $x=y$. Since $S_{m}^{2}(z)=1 /(2-z)$, we have $F=u^{2} S_{m}(z)$ and $F_{u}=2 u S_{m}(z)$. Hence $u=0$. Now we have

$$
F_{z}=\left[S_{m}(z) S_{m-1}(z)+(z-2)\left(S_{m}(z) S_{m-1}(z)\right)^{\prime}\right] x^{2} S_{m-1}(z) .
$$

From $S_{m}^{2}(z)+S_{m-1}^{2}(z)-z S_{m}(z) S_{m-1}(z)=1$ and $S_{m}(z)=S_{m-1}(z)$ we get

$$
(z-2)\left(S_{m}^{\prime}(z)+S_{m-1}^{\prime}(z)\right)=-S_{m}(z) .
$$

It follows that $F_{z}=0$. We have

$$
F_{w}=\left[(2 m+1)+(z-4) S_{m}(z) S_{m-1}(z)+(z-2)\left(T_{m} T_{m-1}\right) w\right] x^{2} S_{m-1}(z) .
$$

From $T_{m}^{2}+w^{2} T_{m-1}^{2}-z T_{m} T_{m-1}=w^{2 m}$ (by Lemma 7.2) and $S_{m}(z)=S_{m-1}(z)$ we get

$$
(2-z)\left(\left(T_{m}\right)_{w}+\left(T_{m-1}\right)_{w}\right) S_{m}(z)+2 S_{m}^{2}(z)=2 m .
$$

It follows that

$$
(2 m+1)+(z-4) S_{m}(z) S_{m-1}(z)+(z-2)\left(T_{m} T_{m-1}\right)_{w}=1+(z-2) S_{m}^{2}(z)=0 .
$$

Hence $F_{w}=0$. The corresponding singular points are $(1: 1: 0, z: 1)$, where $z$ is a root of $S_{m}(z)-S_{m-1}(z)$.

Finally, assume that $x y=0$ and $S_{m}(z)+S_{m-1}(z)=0$. Similar to the above, the singular points are $(1: 1: 0, z: 1)$, where $z$ is a root of $S_{m}(z)+S_{m-1}(z)$.

Definition 7.6 Let $S=\mathcal{Z}(F) \subset \mathbb{P}^{2} \times \mathbb{P}^{1}$ be the vanishing set of $F$ and $\widetilde{S}$ be the desingularization of $S$. 
Now we determine the degenerate fibers; we determine all $(z: w) \in \mathbb{P}^{1}$ such that $F=F_{x}=F_{y}=F_{u}=0$ has at least one solution $(x: y: u) \in \mathbb{P}^{2}$.

Proposition 7.7 The degenerate fibers of $\phi: S \rightarrow \mathbb{P}^{1},(x: y: u, z: w) \mapsto(z: w)$, are

- $\phi^{-1}(1: 0)=\left\{(x: y: u) \in \mathbb{P}^{2} \mid u^{2}=0\right\}$,

- $\phi^{-1}(z: 1)=\left\{(x: y: u) \in \mathbb{P}^{2} \mid u^{2}=0\right\}$, where $z$ is a root of $S_{m-1}(z)$,

- $\phi^{-1}(z: 1)=\left\{(x: y: u) \in \mathbb{P}^{2} \mid\left(x S_{m}(z)-y S_{m-1}(z)\right)\left(y S_{m}(z)-x S_{m-1}(z)\right)=0\right\}$, where $z$ is a root of $S_{3 m}(z)$,

- $\phi^{-1}(z: 1)=\left\{(x: y: u) \in \mathbb{P}^{2} \mid(x-y)^{2}-(2-z) u^{2}=0\right\}$, where $z$ is a root of $S_{m}(z)-S_{m-1}(z)$,

- $\phi^{-1}(z: 1)=\left\{(x: y: u) \in \mathbb{P}^{2} \mid(x+y)^{2}-(2+z) u^{2}=0\right\}$, where $z$ is a root of $S_{m}(z)+S_{m-1}(z)$.

Proof We break the analysis down into cases.

First, we consider the case when $(z: w)=(0: 1)$. We have $F_{x}=F_{y}=0, F=-u^{2}$ and $F_{u}=-2 u$. Hence $u=0$. Note that $\phi^{-1}(1: 0)=\left\{(x: y: u) \in \mathbb{P}^{2} \mid u^{2}=0\right\}$.

Next, we consider the case when $w=1$. First we assume that $S_{m-1}(z)=0$. Then

$$
F_{x}=F_{y}=0, \quad F=-u^{2} S_{m}(z), \quad F_{u}=-2 u S_{m}(z) .
$$

Hence $u=0$. In this case $\phi^{-1}(z: 1)=\left\{(x: y: u) \in \mathbb{P}^{2} \mid u^{2}=0\right\}$.

Finally, we assume that $w=1$ and $S_{m-1}(z) \neq 0$. Note that if $x$ and $y$ are not simultaneously equal to 0 , we must have $S_{m}^{2}(z)-S_{m-1}^{2}(z)=0$.

We first consider the subcase when $x=y=0$, so $(x: y: u)=(0: 0: 1)$. Then

$$
F_{x}=F_{y}=0, \quad F=-S_{3 m}(z), \quad F_{u}=-2 S_{3 m}(z) .
$$

Hence $S_{3 m}(z)=0$. In this case

$$
\phi^{-1}(z: 1)=\left\{(x: y: u) \in \mathbb{P}^{2} \mid\left(x S_{m}(z)-y S_{m-1}(z)\right)\left(y S_{m}(z)-x S_{m-1}(z)\right)=0\right\} .
$$

As a result we may assume that $x y \neq 0$. Therefore

$$
S_{m}(z)-S_{m-1}(z)=0 \quad \text { or } \quad S_{m}(z)+S_{m-1}(z)=0 .
$$

If $S_{m}(z)-S_{m-1}(z)=0$ then $F=F_{x}=F_{y}=F_{u}=0$ is equivalent to $x=y$ and $u=0$. In this case

$$
\phi^{-1}(z: 1)=\left\{(x: y: u) \in \mathbb{P}^{2} \mid(x-y)^{2}-(2-z) u^{2}=0\right\} .
$$


If $S_{m}(z)+S_{m-1}(z)=0$ then $F=F_{x}=F_{y}=F_{u}=0$ is equivalent to $x=-y$ and $u=0$. In this case

$$
\phi^{-1}(z: 1)=\left\{(x: y: u) \in \mathbb{P}^{2} \mid(x+y)^{2}-(2+z) u^{2}=0\right\} .
$$

Next, we consider desingularization. Since $S$ is birational to $\mathbb{P}^{1} \times \mathbb{P}^{1}$, we can blow down $\widetilde{S}$ over $\mathbb{P}^{1}$ some number of times so that it becomes a fiber bundle $\mathbb{P}^{1} \times \mathbb{P}^{1}$ over $\mathbb{P}^{1}$.

Definition 7.8 In the following, let $\chi$ denote the Euler characteristic of a surface. Let $S_{\text {sing }}$ be the set of singular points of $S$ and $N_{\text {sing }}=\left|S_{\text {sing }}\right|$. Furthermore, let $N$ be such that $\widetilde{S}$ is obtained from $\mathbb{P}^{1} \times \mathbb{P}^{1}$ by $N$ one-point blow-ups.

We have

$$
\chi(\tilde{S})=\chi\left(S-S_{\text {sing }}\right)+N_{\text {sing }} \chi\left(\mathbb{P}^{1}\right)=\chi(S)+N_{\text {sing }}
$$

(see [5, Lemma 2.2]).

By definition, $\tilde{S}$ is obtained from $\mathbb{P}^{1} \times \mathbb{P}^{1}$ by $N$ one-point blow-ups. Then since $\chi\left(\mathbb{P}^{1} \times \mathbb{P}^{1}\right)=4$, using $\mathbb{P}^{1} \times \mathbb{P}^{1}$ in place of $S$ in the above, we have

$$
\chi(\tilde{S})=\chi\left(\mathbb{P}^{1} \times \mathbb{P}^{1}\right)+N=4+N .
$$

It follows that $N=\chi(S)+N_{\text {sing }}-4$. We summarize this as a lemma.

Lemma 7.9 We have $N=\chi(S)+N_{\text {sing }}-4$.

Proposition 7.10 The Euler characteristic of $S$ is $\chi(S)= \begin{cases}4+5 m & \text { if } m \geq 1, \\ -5 m & \text { if } m \leq-2 .\end{cases}$

Proof Let $\varphi: S \hookrightarrow \mathbb{P}^{2} \times \mathbb{P}^{1} \rightarrow \mathbb{P}^{1} \times \mathbb{P}^{1}$ be the rational map defined by

$$
(x: y: u, z: w) \mapsto(x: y, z: w) .
$$

Let $P$ be the set of points $(0: 0: 1, z: 1)$ where $z$ is a root of $S_{3 m}(z)$. The map $\varphi$ is not defined at points in $P$. Let $U:=S-P$. We now determine $\varphi(U)$.

Write $F=G+u^{2} H$, where

$$
\begin{aligned}
& G=\left(x y w^{2 m+1}+\left(x y z w-x^{2} w^{2}-y^{2} w^{2}\right) T_{m} T_{m-1}\right) T_{m-1}, \\
& H=\left(-z w^{2 m}+\left(4 w^{2}-z^{2}\right) T_{m} T_{m-1}\right) T_{m-1}-w^{2 m} T_{m} .
\end{aligned}
$$


Note that $\varphi(U)$ is the collection of all points $(x: y, z: w) \in \mathbb{P}^{1} \times \mathbb{P}^{1}$ except those for which $F(x: y, z: w) \in \mathbb{C}[u]$ is a nonzero constant. The polynomial $F(x: y, z: w) \in \mathbb{C}[u]$ is a nonzero constant whenever $H=0$ and $G \neq 0$, which is equivalent to

$$
w=1, \quad S_{3 m}(z)=0 \quad \text { and } \quad\left(x S_{m}(z)-y S_{m-1}(z)\right)\left(y S_{m}(z)-x S_{m-1}(z)\right) \neq 0 .
$$

Hence $\varphi(U)=\mathbb{P}^{1} \times \mathbb{P}^{1}-Q$, where $Q$ is the set of points $(x: y, z: 1) \in \mathbb{P}^{1} \times \mathbb{P}^{1}$ satisfying $S_{3 m}(z)=0$ and

$$
\left(x S_{m}(z)-y S_{m-1}(z)\right)\left(y S_{m}(z)-x S_{m-1}(z)\right) \neq 0 .
$$

Note that $\chi(Q)=0$.

Let $L$ be the set of points $(x: y, z: 1) \in \mathbb{P}^{1} \times \mathbb{P}^{1}$ satisfying $S_{3 m}(z)=0$ and

$$
\left(x S_{m}(z)-y S_{m-1}(z)\right)\left(y S_{m}(z)-x S_{m-1}(z)\right)=0 .
$$

Note that $\{G=H=0\} \subset \mathbb{P}^{1} \times \mathbb{P}^{1}$ is equal to $L$. Hence

Recall that

$$
\chi(L)=\chi\left(\varphi^{-1}(L)\right)= \begin{cases}6 m & \text { if } m \geq 1, \\ -(6 m+4) & \text { if } m \leq-2 .\end{cases}
$$

$$
G=\left(x y w^{2 m}+\left(x y z-x^{2} w-y^{2} w\right) T_{m} T_{m-1}\right) w T_{m-1} .
$$

Since $T_{m}^{2}+w^{2} T_{m-1}^{2}-z T_{m} T_{m-1}=w^{2 m}$, we have

$$
G=\left(x T_{m}-y w T_{m-1}\right)\left(y T_{m}-x w T_{m-1}\right) w T_{m-1} .
$$

Let $B:=\mathcal{Z}(G)$ be the zero set of $G$ in $\mathbb{P}^{1} \times \mathbb{P}^{1}$. Then $B=B_{1} \cup B_{2} \cup B_{3}$, where

$$
\begin{aligned}
& B_{1}=\mathcal{Z}(w)=\mathbb{P}^{1} \times\{(1: 0)\}, \\
& B_{2}=\mathcal{Z}\left(T_{m-1}\right)=\mathbb{P}^{1} \times\left\{(z: 1) \mid S_{m-1}(z)=0\right\}, \\
& B_{3}=\mathcal{Z}\left(x T_{m}-y w T_{m-1}\right) \cup \mathcal{Z}\left(y T_{m}-x w T_{m-1}\right)
\end{aligned}
$$

are subsets in $\mathbb{P}^{1} \times \mathbb{P}^{1}$.

We have $B_{3}=B_{31} \cup B_{32}$, where

$$
B_{31}=\mathcal{Z}\left(x T_{m}-y w T_{m-1}\right) \quad \text { and } \quad B_{32}=\mathcal{Z}\left(y T_{m}-x w T_{m-1}\right) .
$$

Note that $(x: y, z: w) \in B_{31} \cap B_{32}$ if and only if $x=y$ and $T_{m}=w T_{m-1}$, or $x=-y$ and $T_{m}=-w T_{m-1}$. Hence

$$
\begin{aligned}
B_{31} \cap B_{32}=\left\{(1: 1, z: 1) \mid S_{m}(z)-S_{m-1}(z)=0\right\} & \\
& \cup\left\{(1:-1, z: 1) \mid S_{m}(z)+S_{m-1}(z)=0\right\} .
\end{aligned}
$$


It follows that

$$
\chi\left(B_{31} \cap B_{32}\right)= \begin{cases}2 m & \text { if } m \geq 1 \\ -(2 m+2) & \text { if } m \leq-2\end{cases}
$$

Then

$$
\chi\left(B_{3}\right)=\chi\left(B_{31}\right)+\chi\left(B_{32}\right)-\chi\left(B_{31} \cap B_{32}\right)= \begin{cases}4-2 m & \text { if } m \geq 1, \\ 6+2 m & \text { if } m \leq-2 .\end{cases}
$$

We have $B_{1} \cap B_{2}=\varnothing, B_{1} \cap B_{3}=\{(1: 0,1: 0),(0: 1,1: 0)\}$, and

$$
B_{2} \cap B_{3}=\left\{(1: 0, z: 1),(0: 1, z: 1) \mid S_{m-1}(z)=0\right\} .
$$

Hence

$$
\begin{aligned}
& \chi(B)=\chi\left(B_{1}\right)+\chi\left(B_{2}\right)+\chi\left(B_{3}\right)-\chi\left(B_{1} \cap B_{2}\right)-\chi\left(B_{1} \cap B_{3}\right) \\
& -\chi\left(B_{2} \cap B_{3}\right)+\chi\left(B_{1} \cap B_{2} \cap B_{3}\right) \\
& = \begin{cases}2+(2 m-2)+(4-2 m)-0-2-(2 m-2)+0=4-2 m & \text { if } m \geq 1, \\
2-(2 m+2)+(6+2 m)-0-2+(2 m+2)+0=6+2 m & \text { if } m \leq-2 .\end{cases}
\end{aligned}
$$

It follows that

$$
\begin{aligned}
\chi(U) & =2 \chi\left(\mathbb{P}^{1} \times \mathbb{P}^{1}-(B \sqcup Q)\right)+\chi(B-L)+\chi\left(\varphi^{-1}(L)\right) \\
& =2 \chi\left(\mathbb{P}^{1} \times \mathbb{P}^{1}\right)-\chi(B)-2 \chi(Q)-\chi(L)+\chi\left(\varphi^{-1}(L)\right) \\
& = \begin{cases}4+2 m & \text { if } m \geq 1, \\
2-2 m & \text { if } m \leq-2 .\end{cases}
\end{aligned}
$$

Then

$$
\chi(S)=\chi(U)+\chi(P)= \begin{cases}(4+2 m)+3 m=4+5 m & \text { if } m \geq 1, \\ (2-2 m)-(3 m+2)=-5 m & \text { if } m \leq-2 .\end{cases}
$$

Proposition 7.10 and Proposition 7.5 along with the fact that

$$
N=\chi(S)+N_{\text {sing }}-4
$$

give

$$
N=\chi(S)+N_{\text {sing }}-4= \begin{cases}(4+5 m)+4 m-4=9 m & \text { if } m \geq 1, \\ (-5 m)+(-(2+4 m))-4=-(6+9 m) & \text { if } m \leq-2 .\end{cases}
$$

This calculation completes the proof of Theorem 1.5. 


\section{References}

[1] K L Baker, K L Petersen, Character varieties of once-punctured torus bundles with tunnel number one, Internat. J. Math. 24 (2013) MR3078072

[2] S Boyer, E Luft, X Zhang, On the algebraic components of the $\operatorname{SL}(2, \mathbb{C})$ character varieties of knot exteriors, Topology 41 (2002) 667-694 MR1905834

[3] M Culler, P B Shalen, Varieties of group representations and splittings of 3-manifolds, Ann. of Math. 117 (1983) 109-146 MR683804

[4] R Fricke, Ueber die Theorie der automorphen Modulgruppen, Nachr. Ges. Wiss. Göttingen, Math.-Phys. K1. 1896 (1896) 91-101

[5] S Harada, Canonical components of character varieties of arithmetic two bridge link complements, preprint (2012) arXiv:1112.3441

[6] E Landes, Identifying the canonical component for the Whitehead link, Math. Res. Lett. 18 (2011) 715-731 MR2831837

[7] E Landes, On the canonical components of character varieties of hyperbolic 2-bridge link complements, PhD thesis, University of Texas at Austin (2011) Available at http: //hdl . handle.net/2152/ETD-UT-2011-08-2877

[8] M L Macasieb, K L Petersen, R M van Luijk, On character varieties of two-bridge knot groups, Proc. Lond. Math. Soc. 103 (2011) 473-507 MR2827003

[9] T Ohtsuki, Ideal points and incompressible surfaces in two-bridge knot complements, J. Math. Soc. Japan 46 (1994) 51-87 MR1248091

[10] T Ohtsuki, R Riley, M Sakuma, Epimorphisms between 2-bridge link groups, from: "The Zieschang Gedenkschrift", (M Boileau, M Scharlemann, R Weidmann, editors), Geom. Topol. Monogr. 14 (2008) 417-450 MR2484712

[11] K L Petersen, A W Reid, Gonality and genus of canonical components of character varieties, preprint (2014) arXiv:1408.3665

[12] K Qazaqzeh, The character variety of a family of one-relator groups, Internat. J. Math. 23 (2012) MR2888942

[13] P B Shalen, Representations of 3-manifold groups, from: "Handbook of geometric topology”, (R J Daverman, R B Sher, editors), North-Holland, Amsterdam (2002) 9551044 MR1886685

[14] W P Thurston, The geometry and topology of three-manifolds, lecture notes, Princeton University (1979) Available at http://msri.org/publications/books/gt3m

[15] H-o Tokunaga, H Yoshihara, Degree of irrationality of abelian surfaces, J. Algebra 174 (1995) 1111-1121 MR1337188

[16] A T Tran, The universal character ring of the $(-2,2 m+1,2 n)$-pretzel link, Internat. J. Math. 24 (2013) MR3103879 
[17] H Vogt, Sur les invariants fondamentaux des équations différentielles linéaires du second ordre, Ann. Sci. École Norm. Sup. 6 (1889) 3-71 MR1508833

[18] H Yoshihara, Degree of irrationality of a product of two elliptic curves, Proc. Amer. Math. Soc. 124 (1996) 1371-1375 MR1327053

Department of Mathematics, Florida State University

208 Love Building, 1017 Academic Way, Tallahassee, FL 32306-4510, USA

Department of Mathematical Sciences, The University of Texas at Dallas 800 W Campbell Rd FO 35, Richardson, TX 75080, USA

petersen@math.fsu.edu, att140830@utdallas.edu

Received: 13 November $2014 \quad$ Revised: 16 April 2015 